\title{
EFECTO de LAS VINAZAS SOBRE LA GERMINACIÓN DE SOJA, TRIGO Y QUINOA EN CONDICIONES CONTROLADAS
}

\author{
JUAN A. GONZÁLEZ1', PAOLA LANGUASCO² y FERNANDO E. PRADO²
}

\begin{abstract}
Resumen: Las vinazas, derivadas de la producción de alcohol (etanol), encierran la posibilidad de ser utilizadas como fuente de agua y nutrientes. En este estudio se investigó el efecto de las vinazas sobre la germinación y alargamiento radicular en tres especies: Glycine max ("soja"), Triticum aestivum ("trigo") y Chenopodium quinoa ("quinoa"). Se utilizaron vinazas puras y vinazas diluidas (1:1; 1:5; 1:10 y 1:20) tanto a $\mathrm{pH}$ ácido $(\mathrm{pH} 4,5)$ como a $\mathrm{pH}$ neutro. Los resultados demuestran que las vinazas puras inhiben totalmente la germinación en las tres especies utilizadas. En la dilución más baja (1:1) la germinación de soja y quinoa resultaron nulas mientras que en trigo se atrasó y logro un porcentaje de germinación final del 42\%. En resumen, los resultados demuestran que con vinazas diluidas (1:5; 1:10 o 1:20) la germinación en las tres especies ensayadas supera el 90\% de germinación. Sin embargo, el alargamiento radicular sí resulta afectado y esta afectación es mayor a menor dilución de las vinazas. Se sugiere realizar otros estudios complementarios tanto a nivel de ambientes semicontrolados (invernáculos) como experiencias de campo para evaluar los efectos de las diluciones en los distintos estados fenológicos de las especies estudiadas.
\end{abstract}

Palabras clave: Vinazas, germinación, alargamiento radicular.

\begin{abstract}
Vinasse effects on the germination of soybean, wheat, and quinoa under controlled conditions. Vinasse, derivate from alcohol production (ethanol), has the possibilities of being used as a water and nutrient source. In this first study we researched the vinasse effect on germination and radicle elongation in three species: Glycine max (soybean), Triticum aestivum (wheat), and Chenopodium quinoa (quinoa). Pure or diluted vinasse $(1: 1 ; 1: 5 ; 1: 10$ and $1: 20 \mathrm{v} / \mathrm{v})$ was used experiment in acid $\mathrm{pH}(4.5)$ and in neutral $\mathrm{pH}$ (7.2). Results showed that pure vinasse completely inhibits germination in all the studied species. In the lowest dilution 1:1, soybean and quinoa never germinated. Still, the wheat showed a delay in the germination process and achieved a final percentage of $42 \%$. In the other dilutions $(1: 5 ; 1: 10$ and $1: 20)$ all the species, where both $\mathrm{pH}$ ranges were tested, the final germination exceeded $90 \%$. Albeit, radicle elongation was affected in all the treatments with an increasing effect in lower dilutions. We suggest further complementary studies in semi-controlled environment (greenhouses) as well as field experiments to better assess the dilution effect on the different phenological stages of the studied species.
\end{abstract}

Key words: Vinasse, germination, radical elongation.

\section{INTRODUCCIÓN}

Uno de los subproductos de la obtención del alcohol (etanol), a partir de la melaza de la caña de azúcar, son las denominadas vinazas. Estas poseen un $\mathrm{pH}$ bajo (entre 4 y 5), elevada carga orgánica (lo que implica una Demanda Bioquímica

${ }^{1}$ Fundación Miguel Lillo. Instituto de Ecología. Miguel Lillo 251, 4000, Tucumán.

${ }^{2}$ Facultad de Cs. Naturales e IML. Cátedra de Fisiología Vegetal. Miguel Lillo 205, 4000, Tucumán. de Oxígeno, $\mathrm{DBO}_{5}$ entre 40.000 y $60.000 \mathrm{mg}$ $\left.\mathrm{O}_{2} / \mathrm{L}\right)$ y alta concentración del ión potasio $\left(\mathrm{K}^{+}\right)$. Otro problema asociado a las vinazas es el gran volumen que se genera. En efecto, por cada litro de alcohol producido se generan entre 10 a 13 litros de vinazas. Así, una destilería que produce $100 \mathrm{~m}^{3}$ de etanol por día, genera entre 1.000 a $1.300 \mathrm{~m}^{3}$ (o 1.000.000 a 1.300.000 litros) de vinazas. Si se tiene en cuenta que la provincia de Tucumán, una de las mayores productoras de alcohol en el país, produjo aproximadamente $150.000 \mathrm{~m}^{3}$ de etanol en la zafra 2006, un cálculo sencillo demuestra que también se generaron entre 1.500 .000 a $1.950 .000 \mathrm{~m}^{3}$ de 
vinazas. Aunque 13 a 1 es la media más aceptada, la misma puede variar entre 10 a 1 y 15 a 1 (Ferreira \& Montenegro, 1987) según la variedad de la caña utilizada, el tipo de suelo donde se la cultiva, proceso utilizado, entre otras variables. De manera que el tema del volumen de vinazas generado es alto y este podría incrementarse teniendo en cuenta la promoción a la producción de etanol para los denominados biocombustibles.

De manera que, si se tiene en cuenta la elevada carga orgánica de las vinazas, la alta concentración de potasio, su pH ácido y el volumen que se genera anualmente, y que podría incrementarse en los próximos años, arrojar las vinazas a los cursos de agua (arroyos y ríos), sin ningún tipo de tratamiento, representa un peligro potencial para los ecosistemas, especialmente los acuáticos, pues en contacto con el agua puede agotar el oxígeno disuelto, alterar las propiedades físico-químicas y biológicas de los cursos de agua y alterar su composición biótica más la producción de olor.

Sin embargo, también se debe analizar el probable reuso de las vinazas, bajo la perspectiva de su constitución. En efecto, desde un punto de vista físico-químico las vinazas contienen un $9,1 \%$ de materia sólida total mientras que el $90,9 \%$ es agua (datos propios). La materia sólida contiene a su vez un $65 \%$ de materia orgánica y un $35 \%$ de materia inorgánica (Romero Salamanca, 2008; Urbano Terrón, 2002). Los compuestos inorgánicos presentes son el potasio (K), azufre (S), magnesio $(\mathrm{Mg})$, nitrógeno $(\mathrm{N})$ y calcio $(\mathrm{Ca})$. Entre los orgánicos se hallan algunas hexosas (como glucosa y fructosa), varios ácidos (como aconítico, láctico, fumárico, málico, succínico o cítrico, fórmico, butírico, entre otros). Bajo esta óptica algunos autores han propuesto diversos reusos para las vinazas, como por ejemplo, uso para fertiriego del mismo cañaveral (Scandaliaris et al., 1985; Subiros \& Molina, 1992; Dantur et al., 1996; Fadda \& Morandini, 2007), obtención de energía calórica a partir de su concentración (Cárdenas et al., 1986; Cortez \& Brossard Perez, 1997), obtención de energía y recuperación del potasio (Perera, 2009), mezclas con otros residuos para la generación de alimento para ganado (Loaiza de la Pava, 2008), mezclas para la fabricación de compost (Madejón, 1996), entre otros.

Sin embargo, también se debe evaluar la posibilidad de reutilizar las vinazas, puras o diluidas, para el fertiriego de especies cultivadas, sobre todo en aquellos lugares donde el agua es limitante, o bien para la recuperación de especies forestales autóctonas (González \& Prado, no publicado). Esta última perspectiva es bastante interesante desde el punto de vista ambiental pues permitiría el aprovechamiento de las vinazas, o sea grandes volúmenes de agua y los nutrientes asociados, la recuperación de terrenos sin uso como por ejemplo en la zona chaqueña del noroeste de Argentina (NOA) y la consiguiente creación de sumideros de anhídrido carbónico. El conjunto de estas acciones contribuiría fuertemente a disminuir un problema ambiental, como son las vinazas, y por otro lado, permitiría la recuperación de suelos sin usos, la recuperación de especies (de interés económico o autóctonas de interés ambiental) y la creación de sumideros de carbono.

Sin embargo, estas perspectivas de aprovechamiento de vinazas, requiere de estudios, de laboratorio y de campo, que proporcionen información cierta sobre este residuo y sus efectos. Bajo esta perspectiva se ha comenzado a estudiar las vinazas y sus efectos sobre la primera etapa de establecimiento de una especie y que es la germinación. El objetivo de este estudio es estudiar el efecto de vinazas, en distintos grados de dilución, sobre la germinación y elongación radicular de tres especies graníferas dos de las cuales se cultivan ya en Tucumán y otra de potencial cultivo en la zona como un primer paso en el estudio de reaprovechamiento de este residuo industrial.

\section{Materiales y Métodos}

Vinazas utilizadas: en todos los ensayos se utilizaron vinazas provenientes de un ingenio localizado en el Departamento Cruz Alta (Tucumán). Estas fueron tomadas a la salida del proceso de destilación de alcohol donde se midieron algunas variables físico - químicas como $\mathrm{pH}$, conductividad eléctrica, temperatura, oxígeno disuelto y salinidad. Las variables mencionadas fueron medidas con una sonda multiparamétrica previamente calibrada (Water Quality Checker, Horiba, Japan).

Especies utilizadas: Se utilizaron dos especies productoras de grano: trigo (Triticum aestivum) cv Baguette y soja (Glycine max) cv A6126 que son importante cultivos en la zona límite entre 
las provincias de Tucumán y Santiago del Estero (llanura chaqueña). También se utilizó "quínoa" (Chenopodium quinoa) cv CICA como una especie de alta velocidad de germinación en laboratorio donde los efectos de las vinazas pueden hacerse visibles rápidamente.

Ensayos de germinación: Para evaluar el efecto de las diluciones de vinaza sobre la germinación, se empleó un diseño experimental constituido por cuatro (4) tratamientos y un control: (1) Control (con agua destilada); (2) vinaza pura; 3) vinaza 1:1 (v/v); vinaza 1:5 (v/v), (3) vinaza 1:10 (v/v) y vinaza 1:20 (v/v). Se realizaron 5 réplicas por tratamiento y todos los ensayos se repitieron tres veces. Previo a los ensayos de germinación, las semillas se esterilizaron con hipoclorito de sodio al $2 \%$ durante 3 minutos y 3 lavados posteriores con agua destilada. Esto tuvo como objetivo evitar el ataque de hongos. Todos los ensayos se realizaron en caja de Petri de 5 a $9 \mathrm{~cm}$ de diámetro a las que se les colocaba un papel de filtro que posteriormente era embebido con la solución de vinaza a ensayar. El volumen de vinaza agregado a cada caja de Petri varió de acuerdo al diámetro de la misma y al tamaño de la semilla a evaluar. Dicho volumen osciló entre $2 \mathrm{ml}$ (en el caso de "quínoa") y $5 \mathrm{ml}$ (soja y trigo). Las cajas fueron puestas a germinar en una habitación con control de temperatura $\left(25^{\circ} \mathrm{C}\right)$. Como germinadas fueron consideradas todas aquellas semillas con una radícula $\geq 2 \mathrm{~mm}$; mientras que semillas abortadas (germinación anormal) fueron consideradas aquellas que presentaron emergencia de cotiledones o hipocótilo previo a la radícula. El número de semillas germinadas y/o abortadas se registró cada $2 \mathrm{~h}$ durante $36 \mathrm{~h}$ para quínoa, $62 \mathrm{~h}$ para trigo y $96 \mathrm{~h}$ para soja. Estos valores se establecieron en función del tiempo requerido por cada especie para alcanzar su porcentaje máximo de germinación.

Efecto de la acidez de la vinaza sobre la germinación: como la vinaza posee un $\mathrm{pH}$ muy bajo se realizaron experiencias para verificar que grado de influencia posee este $\mathrm{pH}$ sobre la germinación. Para ello, se realizó un primer ensayo de germinación (como se describe Ensayos de germinación) con la vinaza diluida con el $\mathrm{pH}$ intrínseco $(4,7$ a 4,9$)$ y otro con $\mathrm{pH}$ ajustado a 7,2. Esto último se logro con adicción de $\mathrm{NaOH} 0,2 \mathrm{~N}$.

Medición de longitud y diámetro radicular: Posterior al conteo de las semillas germinadas se procedió a separar 20 de ellas de cada caja de Petri colocándolas en tubos de hemólisis conteniendo etanol $96 \%$. Esto se realizó a fin de conservar las radículas en óptimas condiciones para su posterior medición de longitud y diámetro radicular. Para esto se utilizó un calibre digital (Digimess) en milímetros con una precisión de dos decimales. El diámetro se midió a la altura media, establecida en base a la longitud de cada radícula en particular.

Análisis de datos: Los resultados obtenidos se analizaron estadísticamente mediante el análisis de la varianza (con $p \leq 0,05)$ usando el programa estadístico InfoStat (2001). La comparación estadística entre los tratamientos se realizó mediante el test de Tukey. A efectos de comparar el nivel de germinación alcanzado en cada tratamiento por las especies estudiadas, se utilizaron los datos correspondientes a la máxima germinación de cada una de ellas que fueron: $24 \mathrm{~h}$ para quinoa, $50 \mathrm{~h}$ para trigo y $80 \mathrm{~h}$ para soja.

\section{Resultados}

\section{Vinaza utilizada}

Las propiedades fisicoquímicas de la vinaza utilizada muestran en la Tabla 1.

\section{Germinación}

\section{Chenopodium quinoa ("quinoa")}

En general, la germinación en las distintas concentraciones, tanto a $\mathrm{pH}$ ácido como a $\mathrm{pH}$ ajustado (Fig. 1A, B) sigue un modelo logístico. Las distintas diluciones sólo retrasan la misma pero en ningún caso se llega a la inhibición. Todos los tratamientos muestran valores de germinación por arriba del $90 \%$. En la gráfica no se consignan los valores de germinación en los tratamientos con vinaza pura y vinaza 1:1 debido a que la germinación fue nula en ambos $\mathrm{pH}$.

Tabla 1. Parámetros fisicoquímicos de las vinazas utilizadas en los ensayos.

\begin{tabular}{|cccccc|}
\hline $\mathrm{pH}$ & $\begin{array}{c}\mathrm{C} . \mathrm{E} . \\
(\mathrm{mS} / \mathrm{cm})\end{array}$ & $\begin{array}{c}\text { Turb. } \\
(\mathrm{NTU})\end{array}$ & $\begin{array}{c}\text { OD } \\
(\mathrm{mg} \text { O2/l) }\end{array}$ & $\begin{array}{c}\mathrm{T} \\
\left({ }^{\circ} \mathrm{C}\right)\end{array}$ & $\begin{array}{c}\text { Salinidad } \\
(\%)\end{array}$ \\
\hline 4,8 & 40,9 & 999 & 0,19 & 90 & 2,6 \\
\hline
\end{tabular}

C.E.: Conductividad eléctrica, Turb: turbidez, OD: oxígeno disuelto, T: temperatura. 
Bol. Soc. Argent. Bot. 49 (4) 2014
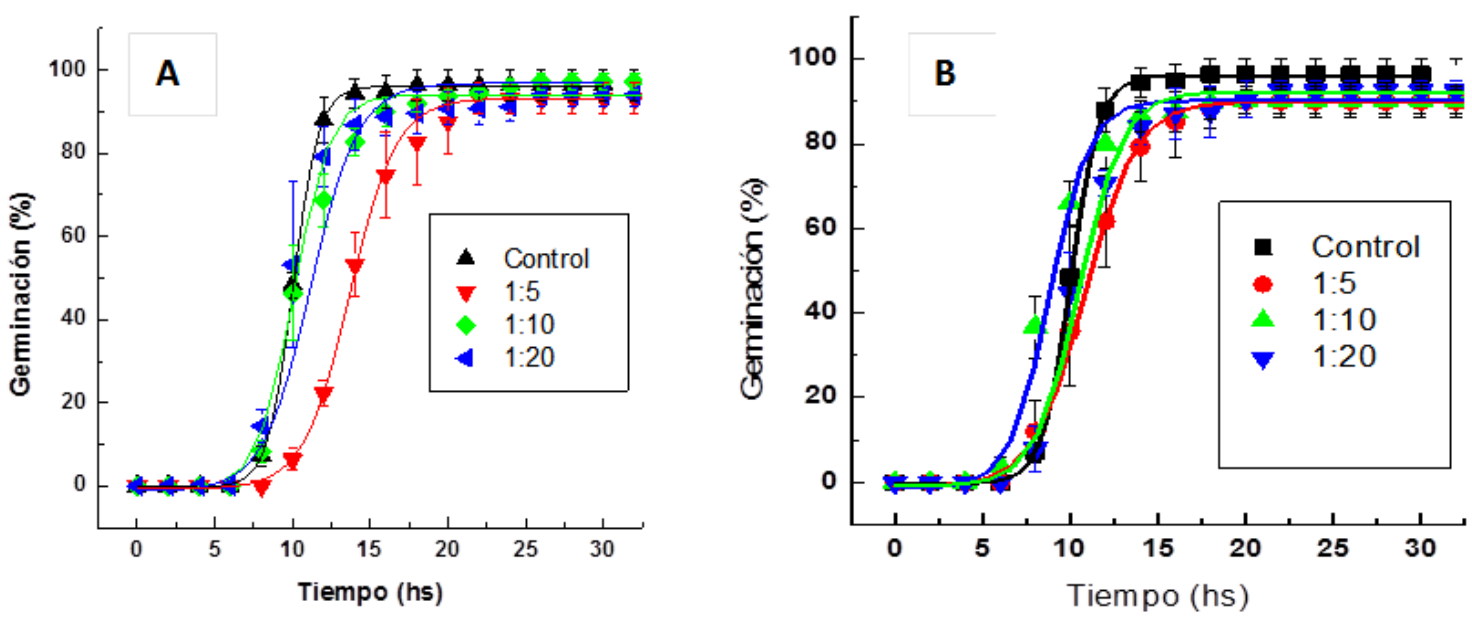

Fig. 1. Chenopodium quinoa. Efecto de distintas concentraciones de vinaza a pH ácido (A) y pH ajustado (B) sobre la germinación. Cada punto es el promedio de tres experiencias distintas con 5 repeticiones cada una. La barra vertical representa la desviación estándar.

En la Tabla 2 se resumen otras variables relacionadas con la germinación máxima $\left(\mathrm{G}_{\max }\right)$, tiempo para llegar al máximo de germinación $\left(\mathrm{t}_{\max }\right)$ y tiempo para llegar a una germinación del $50 \%$ $\left(\mathrm{G}_{50}\right)$.

La germinación máxima disminuye en todos los tratamientos ya sea con $\mathrm{pH}$ ácido o con el mismo ajustado a 7,2. El mayor efecto sobre la $\mathrm{G}_{\max }$ se registra a $\mathrm{pH} 7$, donde la germinación disminuye de $96,4 \%$ a $90 \%$ en la dilución $1: 5$; en cambio en $\mathrm{pH}$ ácido y en esta misma dilución disminuye de $96,4 \%$ a $93,2 \%$. Los datos en general indican que la dilución 1:10 y a $\mathrm{pH}$ ácido es la menos severa ya que supera incluso al control. Este hecho no se verifica a $\mathrm{pH}$ ajustado. Por otro lado, el tiempo para llegar al máximo de germinación $\left(\mathrm{t}_{\max }\right)$ aumenta a medida que la dilución es mayor.

Tabla 2. Chenopodium quinoa. Germinación máxima $\left(G_{\max }\right)$, tiempo para llegar al máximo de germinación $\left(\mathrm{t}_{\max }\right)$ y tiempo para llegar a una germinación del $50 \%\left(\mathrm{G}_{50}\right)$.

\begin{tabular}{|lccccccc|}
\hline & \multicolumn{3}{c}{ pH ácido } & \multicolumn{3}{c|}{ pH ajustado } \\
& $\begin{array}{c}\text { Gmax } \\
\text { (\%) }\end{array}$ & $\begin{array}{c}\text { Tmax } \\
\text { (hs) }\end{array}$ & $\begin{array}{c}\text { G50 } \\
\text { (hs) }\end{array}$ & $\begin{array}{c}\text { Gmax } \\
\text { (\%) }\end{array}$ & $\begin{array}{c}\text { Tmax } \\
\text { (hs) }\end{array}$ & $\begin{array}{c}\text { G50 } \\
\text { (hs) }\end{array}$ \\
\hline Control & 96,4 & 18 & 10 & 96,4 & 18 & 10 \\
'1:5 & 93,2 & 22 & 13,7 & 90 & 20 & 10,8 \\
'1:10 & 97,2 & 26 & 11,2 & 90,4 & 20 & 8,8 \\
'1:20 & 94 & 26 & 10,1 & 92,4 & 22 & 10,5 \\
\hline
\end{tabular}

Esto hecho se verifica tanto a $\mathrm{pH}$ ácido como a $\mathrm{pH}$ ajustado aunque la magnitud cambia (Tabla 2). Sin embargo, debe mencionarse que tanto el $\mathrm{t}_{\max }$ como $\mathrm{G}_{50}$ son menores en $\mathrm{pH}$ ajustado que en $\mathrm{pH}$ ácido.

\section{Glycine max ("soja")}

También en este caso las vinazas, a pH ácido o ajustado, sólo retrasa la germinación de soja en el tiempo (Fig. 2A, B). A pH ácido el mayor efecto se registra en la dilución 1:5 donde la germinación máxima llega a $94,8 \%$ contra $98,4 \%$ del control. A pH ajustado la germinación es mayor $(99,2 \%)$ y de nuevo el efecto más pronunciado es en la dilución 1:5 donde se llega a una germinación máxima de $97,2 \%$. También en este caso no se adjuntan los datos de germinación en los tratamientos con vinaza pura y 1:1 debido a que la misma fue cero en todo el tiempo considerado y en ambos $\mathrm{pH}$.

El tiempo para llegar a la máxima germinación $\left(\mathrm{G}_{\max }\right)$ es afectado de manera distinta según sea el $\mathrm{pH}$ (Tabla 3). En efecto, a $\mathrm{pH}$ ácido el $\mathrm{t}_{\max }$ se incrementa con respecto a los que se registra a pH ajustado. Es evidente que la acidez, inherente a la vinaza, produce un efecto de retardo en la germinación. Este retardo, comparado al control, es más pronunciado a dilución 1:5 (72 a 92 hs) que a dilución 1:20 (72 a 80 hs). En cambio a pH ajustado los $\mathrm{t}_{\max }$ también aumentan con respecto al control pero son menores comparados a los de $\mathrm{pH}$ ácido. Este efecto, se refleja bien en el $\mathrm{G}_{50}$ (Tabla 3). 

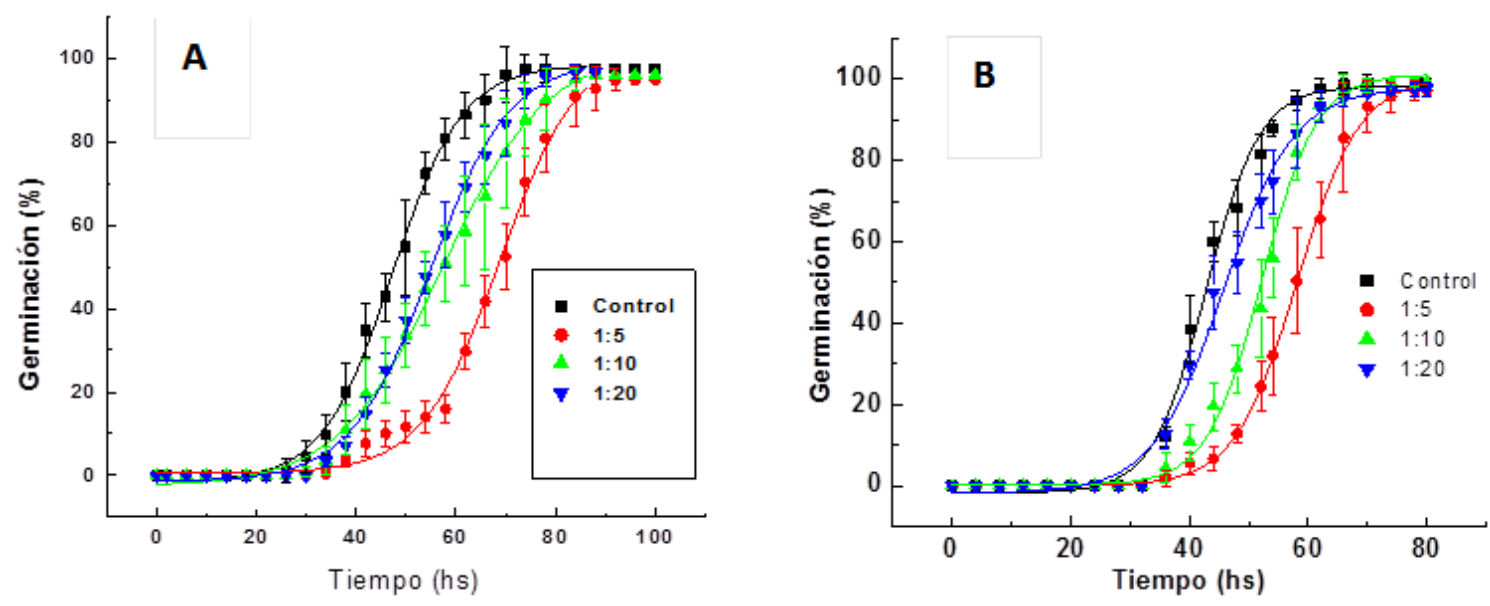

Fig. 2. Glycine max. Efecto de distintas concentraciones de vinaza a pH ácido (A) y pH ajustado (B) sobre la germinación. Cada punto es el promedio de tres experiencias distintas con 5 repeticiones cada una. La barra vertical representa la desviación estándar.

\section{Triticum aestivum ("trigo")}

En ambos pH (ácido y ajustado a 7) la germinación final varía entre 98,8 y $100 \%$ y 98,4 y $100 \%$ respectivamente (Fig. 3A, B). El mayor efecto de retardo en la germinación se produce en la dilución 1:5. Mientras a $\mathrm{pH}$ ácido el tiempo para llegar a la máxima germinación es de 66 horas a pH ajustado a 7 el tiempo se reduce a 62 horas. No se muestran los datos referidos al tratamiento con vinaza pura pues la germinación en este caso fue nula. Sin embargo, en el tratamiento 1:1 a partir de la hora 50 la germinación se activa y alcanza un valor máximo del $42 \%$ en la hora 70 y luego se estabiliza durante el resto del tiempo.

En relación al tiempo para llegar al 50\% de la germinación el mismo se incrementa más en $\mathrm{pH}$ ácido que en $\mathrm{pH}$ ajustado con un efecto mas pronunciado

Tabla 3. Glycine max. Germinación máxima $\left(\mathrm{G}_{\max }\right)$, tiempo para llegar al máximo de germinación $\left(\mathrm{t}_{\max }\right)$ y tiempo para llegar a una germinación del $50 \%\left(\mathrm{G}_{50}\right)$.

\begin{tabular}{|llllllll|}
\hline & \multicolumn{3}{c}{ pH ácido } & \multicolumn{3}{c|}{ pH ajustado } \\
& $\begin{array}{c}\text { Gmax } \\
\text { (\%) }\end{array}$ & $\begin{array}{c}\text { Tmax } \\
\text { (hs) }\end{array}$ & $\begin{array}{c}\text { G50 } \\
\text { (hs) }\end{array}$ & $\begin{array}{c}\text { Gmax } \\
\text { (\%) }\end{array}$ & $\begin{array}{c}\text { Tmax } \\
\text { (hs) }\end{array}$ & $\begin{array}{c}\text { G50 } \\
\text { (hs) }\end{array}$ \\
\hline Control & 98,4 & 72 & 47,3 & 98,4 & 72 & 47,3 \\
'1:5 & 94,8 & 92 & 69 & 97,2 & 78 & 58,1 \\
'1:10 & 96 & 88 & 57,3 & 99,2 & 74 & 52,2 \\
'1:20 & 96,8 & 80 & 54,7 & 97,6 & 76 & 45,7 \\
\hline
\end{tabular}

en la dilución 1:5 (Tabla 4). A medida que aumenta la dilución de la vinaza los valores de $\mathrm{G}_{50}$ se aproximan más al control en $\mathrm{pH}$ ajustado que en $\mathrm{pH}$ ácido.

\section{Longitud y diámetro radicular}

Los datos de las figuras 4,5 y 6 muestran que tanto las vinazas con $\mathrm{pH}$ ácido como en aquellas que se ajusto el mismo a 7 producen una fuerte disminución en el alargamiento de las radículas. Este efecto esta disminución aumenta a medida que la dilución es menor. Por ejemplo, las vinazas ácidas, en dilución $1: 5$, provocan una disminución del alargamiento radicular del 34\% en "trigo", 50\% en "quinoa" y $56 \%$ en "soja": Estos valores se incrementan más aún cuando el $\mathrm{pH}$ de las vinazas es ajustado.

La dilución que menos afecta la elongación radicular, tanto a $\mathrm{pH}$ ácido como a $\mathrm{pH}$ ajustado,
Tabla 4. Triticum aestivum. Germinación máxima $\left(G_{\max }\right)$, tiempo para llegar al máximo de germinación $\left(\mathrm{t}_{\max }\right)$ y tiempo para llegar a una germinación del $50 \%\left(\mathrm{G}_{50}\right)$.

\begin{tabular}{|lccccccc|}
\hline & \multicolumn{3}{c}{ pH ácido } & \multicolumn{3}{c|}{ pH ajustado } \\
& $\begin{array}{c}\text { Gmax } \\
\text { (\%) }\end{array}$ & $\begin{array}{c}\text { Tmax } \\
\text { (hs) }\end{array}$ & $\begin{array}{c}\text { G50 } \\
\text { (hs) }\end{array}$ & $\begin{array}{c}\text { Gmax } \\
\text { (\%) }\end{array}$ & $\begin{array}{c}\text { Tmax } \\
\text { (hs) }\end{array}$ & $\begin{array}{c}\text { G50 } \\
\text { (hs) }\end{array}$ \\
\hline Control & 100 & 54 & 28,6 & 100 & 54 & 28,6 \\
'1:5 & 100 & 66 & 40,5 & 99,2 & 62 & 33,4 \\
'1:10 & 98,8 & 58 & 32,3 & 100 & 58 & 27,6 \\
'1:20 & 99,2 & 54 & 30,1 & 98,4 & 42 & 27,2 \\
\hline
\end{tabular}



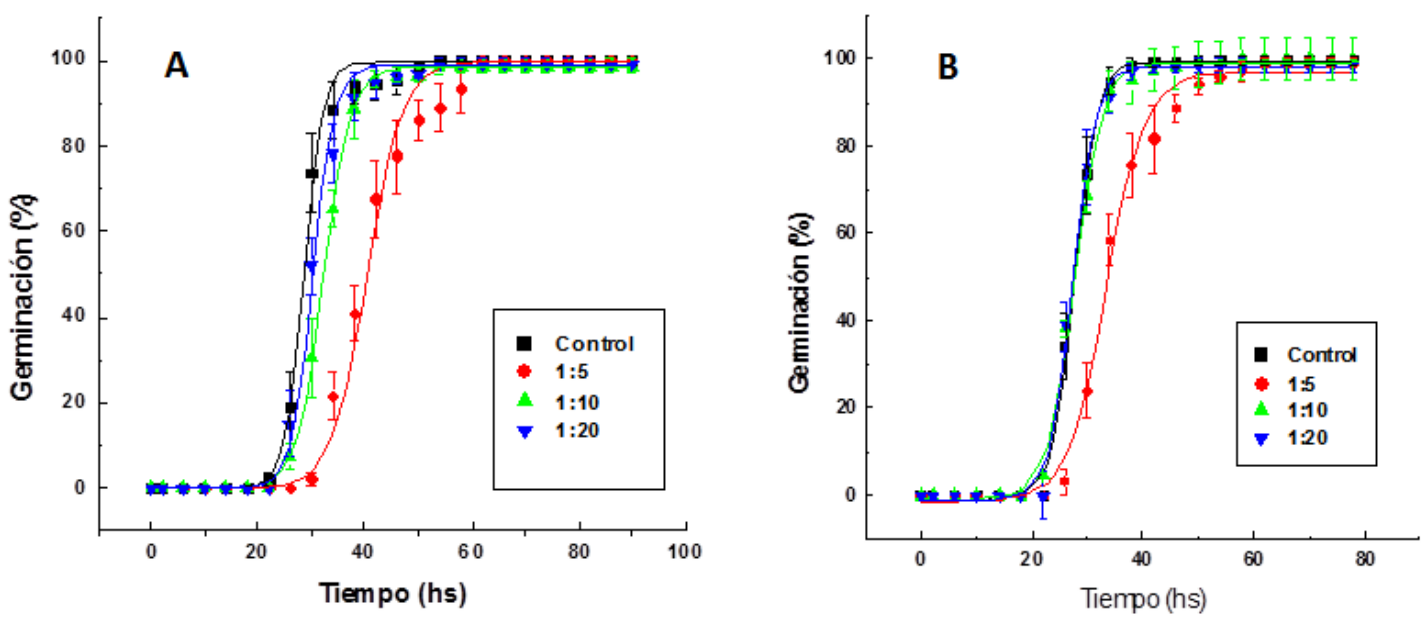

Fig. 3. Triticum aestivum. Efecto de distintas concentraciones de vinaza a pH ácido (A) y pH ajustado (B) sobre la germinación. Cada punto es el promedio de tres experiencias distintas con 5 repeticiones cada una. La barra vertical representa la desviación estándar.

es la correspondiente a 1:20. Sin embargo, debe mencionarse que G. max sigue siendo la más afectada y en este caso con porcentajes de disminución en el crecimiento que oscilan entre $36,1 \%$ y $41,8 \%$ según el pH sea ácido o ajustado respectivamente.

El diámetro radicular resulta afectado según la dilución, $\mathrm{pH}$ considerado y especie (Tabla 5). En el caso de quinoa en $\mathrm{pH}$ ácido no existen diferencias significativas $(\mathrm{p}<0,05)$ entre los tratamientos con el control. Sin embargo, en $\mathrm{pH}$ neutro aparecen

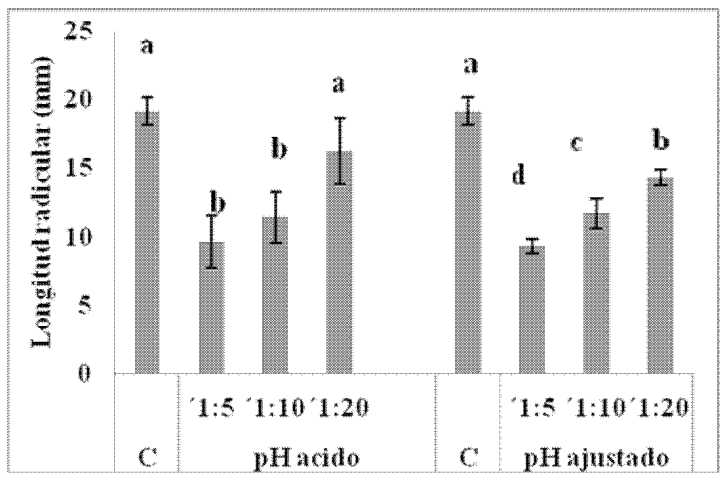

Fig. 4. Chenopodium quinoa. Efecto de las vinazas sobre la longitud radicular $(\mathrm{mm})$ en $\mathrm{pH}$ ácido y $\mathrm{pH}$ ajustado luego de 24 horas de incubación a $25^{\circ} \mathrm{C}$. Las barras verticales representan el valor promedio de 100 plantas con su desviación estándar. Barras con la misma letra no son significativamente diferentes $(p<0.05)$. C: Control. diferencias destacándose aquel diámetro en dilución $1: 10$ que resulta ser un $23,7 \%$ mayor que el control. En el caso de la soja el diámetro radicular disminuye con respecto al control en todos los tratamientos. El efecto de disminución es mayor en las concentraciones que poseen mayor cantidad de vinazas (1:5 en este caso). Por su lado, en el trigo el diámetro en todos los tratamientos resulta afectado hacia la disminución pero estas diferencias no son significativas $(\mathrm{p}<0,05)$.

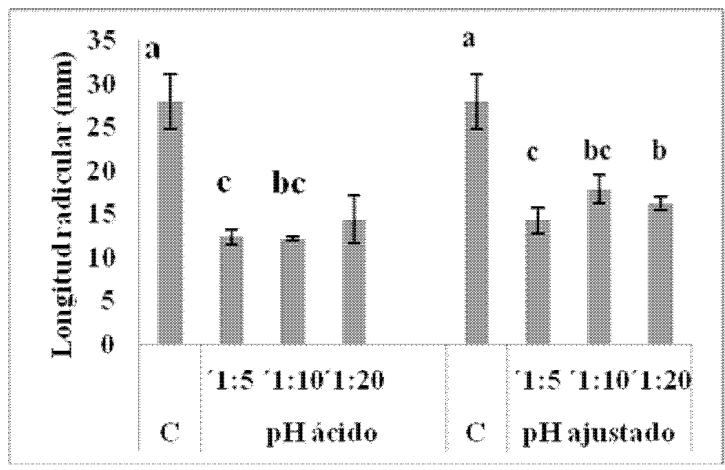

Fig. 5. Glycine max. Efecto de las vinazas sobre la longitud radicular $(\mathrm{mm})$ en $\mathrm{pH}$ ácido y $\mathrm{pH}$ ajustado luego de 80 horas de incubación a $25^{\circ} \mathrm{C}$. Las barras verticales representan el valor promedio de 100 plantas con su desviación estándar. Barras con la misma letra no son significativamente diferentes ( $p<$ 0.05). C: Control 


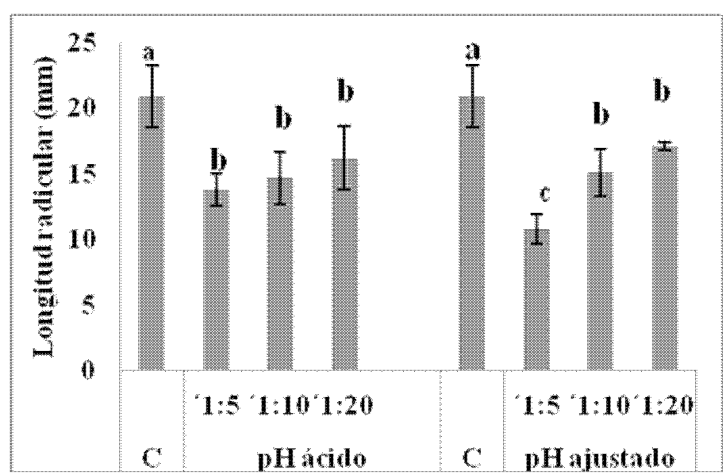

Fig. 6. Triticum aestivum. Efecto de las vinazas sobre la longitud radicular $(\mathrm{mm})$ en $\mathrm{pH}$ ácido y $\mathrm{pH}$ ajustado luego de 50 horas de incubación a $25^{\circ} \mathrm{C}$. Las barras verticales representan el valor promedio de 100 plantas con su desviación estándar. Barras con la misma letra no son significativamente diferentes $(p<0.05)$. C: Control.

\section{Discusıón}

Las vinazas están constituidas en términos generales por un $90 \%$ de agua y un $10 \%$ de sustancias orgánicas e inorgánicas (datos propios no publicados). La carga orgánica que poseen hace que las mismas, si son arrojadas a los cursos de agua sin ningún tipo de tratamiento, agoten el oxígeno del agua, generando una situación delicada para los ambientes acuáticos. Teniendo en cuenta que por cada litro de alcohol (etanol) producido se generan entre 10 a 13 litros de vinazas, el volumen resultante anual de este residuo líquido es importante. Sólo a modo de ejemplo se menciona que para una producción de $150.000 \mathrm{~m}^{3}$ de etanol (que fue lo que se produjo en la zafra 2006 para todo el Noroeste Argentino), también se generaron entre 1.500 .000 a $1.950 .000 \mathrm{~m}^{3}$ de vinazas. Si bien las vinazas no deben eliminarse a los cursos de agua, por los efectos comentados, es necesario estudiar el reuso de las mismas ya que desde el punto de vista ambiental las vinazas son una fuente de agua y materia (orgánica e inorgánica) que pueden ser de utilidad con el tratamiento correspondiente.

Una posibilidad que se hipotetiza para el reuso de las vinazas, es su aplicación en el campo en forma pura o en dilución 1:1 con agua, para irrigar campos previo a la siembra de granos en la zona este (E) de Tucumán o bien para la fertilización o fertiirrigación en caña de azúcar o en otras especies
Tabla 5. Efecto de las vinazas sobre el diámetro radicular $(\mathrm{mm})$ de las tres especies utilizadas.

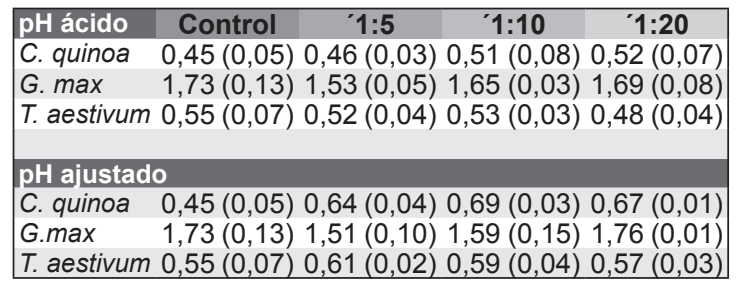

Valor promedio correspondientes a la medición de 100 plántulas la finalizar la experiencia. El valor entre paréntesis corresponde a la Desviación Estándar.

de interés agronómico. Los resultados de nuestra experiencia con soja, trigo y quinoa demuestran que esta práctica sería posible sólo si se tienen en cuenta algunas diluciones con agua. En efecto, sí se utiliza vinaza pura, sin ningún tipo de tratamiento la germinación en las tres especies ensayadas es nula. Cuando la vinaza se diluye en una proporción 1:1 tanto la soja como la quinoa son todavía afectadas sin que se llegue a la germinación propiamente dicha. Sin embargo, en el caso del trigo, se registra un atraso de la germinación hasta la hora 50. A partir de este momento la germinación se activa pero se estabiliza en un porcentaje del $42 \%$ a partir de la hora 70. Teniendo en cuenta que se en este caso si bien se produce germinación hay una pérdida del $58 \%$ de las semillas en la práctica este resultado puede tornarse antieconómico.

Las otras diluciones de vinazas utilizadas en este estudio $(1: 5,1: 10$ y $1: 20)$ por el contrario no inhibieron la germinación de la soja, trigo y quinoa. Así en el caso de quinoa la germinación máxima, en pH ácido, osciló entre 93 y 97\% mientras que en pH ajustado osciló entre 90 y $92 \%$. Si se tiene en cuenta que el control tuvo un porcentaje de germinación del $96,4 \%$ las disminuciones mencionadas no fueron tan pronunciadas.

En el caso de la soja la germinación a $\mathrm{pH}$ ácido osciló entre 94,8 y 96,8\% valores que se incrementaron a 97,2 y $99,2 \%$ al ajustarse el $\mathrm{pH}$. En el caso del trigo no se detectaron diferencias significativas entre los porcentajes de germinación en ambos $\mathrm{pH}$.

En consonancia con nuestro estudio se menciona que investigaciones realizadas en España con semillas de pepino han demostrado 
que la germinación de las mismas no presentaba diferencias significativas entre los distintos tratamientos utilizados (vinazas diluidas al $10 \%, 15 \%$ y $20 \%$ ) y que en todos los casos la germinación obtenida fue superior al 90\% (Santos et al. 2006). Por otro lado, estudios realizados con vinazas diluidas (ver Navarro et al., 2006) han demostrado que la germinación en lechuga, achicoria y escarola se inhibe ( $40 \%$ promedio) en diluciones de $10^{-1}$. Sin embargo, estas inhibiciones se recuperan a medida que las diluciones se hacen mayores $\left(10^{-2}, 10^{-3}\right.$ y $\left.10^{-4}\right)$. Por otro lado, tanto las raíces como los hipocótilos de las mismas especies mencionadas sufren fuertes retardos en su crecimiento en las diluciones utilizadas pero el efecto más pronunciado lo registraron entre $10^{-1}$ y $10^{-2}$ (Navarro et al., 2006). Sin embargo, debe apuntarse que tanto las diluciones como las especies utilizadas, son diferentes a las utilizadas en nuestro trabajo. Este hecho podría explicar las diferencias puntuales encontradas. Sin embargo, se reafirma nuestro estudio que las vinazas diluidas no inhiben la germinación sino que la retardan y que el crecimiento de las radículas sí resulta afectado. Resta aún estudiar el efecto del riego con vinazas en otras etapas posteriores a la germinación y en especial el efecto sobre el rendimiento de la especie que se considere.

Con respecto al efecto del $\mathrm{pH}$ ácido de las vinazas sobre la germinación y desarrollo de las plántulas, algunos autores (Navarro et al., 2006) propusieron neutralizar la vinaza con $\mathrm{NaOH} 20 \%$ antes de su utilización, argumentando que de lo contrario se podrían producir efectos negativos sobre la germinación y el desarrollo de las plántulas. Nuestros resultados demuestran que tanto a $\mathrm{pH}$ bajos (4,5 en este estudio) como en aquellos cercanos al neutro ( 7,2 en este estudio) la germinación se afecta pero no significativamente con diferencias que no superan el 2-3\%. Si resulta significativamente afectado el alargamiento radicular (en las tres especies ensayadas y en ambos $\mathrm{pH}$ ) lo que podría tener efectos físicos (para el soporte de las plantas por ejemplo) como fisiológicos (para la toma de agua y nutrientes por ejemplo). Por lo tanto, para el estadio de germinación de quinoa, soja y trigo se puede concluir que la acidez de la vinaza diluida 5 veces afecta significativamente el alargamiento radicular pero no así la germinación. Esto abre la posibilidad de utilizar vinazas diluidas con agua industrial (derivadas de los procesos que se utilizan en la fabricación de azúcar y de etanol) para riego de áreas ubicadas en el este (E) de la provincia de Tucumán, que se caracterizan justamente por poseer un déficit de agua en los meses de otoño y primavera. Sin embargo, es necesario profundizar estos estudios para las otras etapas fenológicas de las especies utilizadas y sobre todo evaluar los rendimientos.

En la actualidad se tiende al reuso de las vinazas en dos formas: aplicación de las mismas pura o diluida, o bien como una masa privada de agua. En el primer caso se habla de fertiirrigación y con esta práctica se provee a las planta de agua y nutrientes. En el segundo, donde se han concentrado los nutrientes, se habla de fertilización. Optar por una forma $\mathrm{u}$ otra depende de otras variables, que necesariamente se deben considerar como: período de aplicación, necesidades de agua del cultivo, tipo de suelos y su contenido en potasio, microclima donde se desarrolla el cultivo y estado nutricional de la planta. En forma particular, se debe prestar atención al hecho que desde los últimos días de agosto y aproximadamente hasta la segunda quincena de noviembre las precipitaciones en toda la provincia de Tucumán son escasas, el déficit hídrico es marcado y la necesidad de riego puede ser considerada como permanente. Sin embargo, dada la estacionalidad climática desde la segunda quincena de noviembre en adelante el riego es complementario y los déficit son aleatorios. Cuando se presentan, ocurren en áreas importantes y son muy difíciles de pronosticar con antelación. También se debe tener en cuenta que la napa freática en la provincia es de profundidad variable (Figueroa et al., 1996) y que además el nivel freático fluctúa a lo largo del año registrándose los niveles más altos desde enero hasta abril por efecto de las lluvias de verano, y los más bajos de septiembre a noviembre. De manea que la aplicación de vinaza podría circunscribirse al período setiembre - noviembre pero con varias consideraciones. Una de ellas es que las vinazas poseen una alta concentración de potasio (K). Por lo tanto, será necesario evaluar la concentración de potasio en los diferentes lugares de cultivo en la provincia, la sensibilidad de los cultivos a irrigar y establecer las dosis máximas a aplicar según el suelo y cultivo a ensayar. Además 
se deberá considerar el efecto de un probable exceso de potasio sobre las características físicas, químicas y biológicas del suelo.

\section{Agradecimientos}

Este trabajo se realizó dentro del proyecto "Efecto de las vinazas sobre las primeras etapas de germinación y plántulas en especies nativas e introducidas" en el Instituto de Ecología de la Fundación Miguel Lillo. Se agradece al Ing. H. Alemanny quién nos proporcionó las semillas de trigo y soja.

\section{Bibliografía}

CÁRDENAS, G. E. J. YOCCA \& J. C. D. LOZANO. 1986. Determinación del calor de combustión en vinazas. Balance energético del proceso global evaporación-incineración. Revista Ind. Agric. Tucumán 63: 79-99.

CORTEZ, L. A. B \& L. E. BROSSARD PÉREZ. 1997. Experiences on vinasse disposal part III: Combustion of vinasse- \# 6 fuel oil emulsions. Brazilian J. Chem. Engin. 14 (1). Sin páginas. Consulta http://www.scielo.br/scielo.php?script=sci_ arttext\&pid=S0104-66321997000100002

DANTUR, A., J. SCANDALIARIS, F. PÉREZZAMORA \& M. A. RONCEDO. 1996. Aprovechamiento agrícola de los residuos de la agroindustria de la caña de azúcar. Parte II. El uso de la vinaza. Avance Agroindustrial 16:38-41.

FADDA, G. \& M. MORANDINI. 2007. El uso agrícola de la vinaza. Revisión de antecedentes y caracterización de las condiciones del área cañera tucumana para su aplicación. Publicación Especial $N^{\circ} 33$. Estación Experimental Agroindustrial Obispo Colombres, Tucumán.

FERREIRA, S. E. \& MONTENEGRO, O. A.1987. Efeitos da aplicacao da vinhaça nas propriedades químicas, físicas e biológicas do solo. Boletín Técnico COPERSUCAR 36: 1-7.
FIGUEROA, L. R, L. F. MEDINA\&A. M. PIETROBONI. 1996. Variaciones del nivel freático en la llanura deprimida de Tucumán. Serie Monográfica. INTACRTS, Tucumán.

LOAIZA DE LA PAVA, K. J. 2008. Usos de los subproductos de la agroindustria de la caña en la elaboración de dos suplementos nutricionales para rumiantes en el valle de Cauca. Tesis Doctoral. Universidad de Caldas (Colombia). En: Leal, R.H. 2013. Conversión de la cachaza azucarera en coproductos de valor agregado para la alimentación de ganado bovino en el marco de un desarrollo sustentable. Tesis de Maestría, Instituto Politécnico Nacional, México).

MADEJÓN, E. 1996. Aprovechamiento agronómico de compost de vinaza: efectos en suelos, cultivos y aguas de drenaje. Tesis Doctoral, Universidad de Sevilla. 260 páginas.

PERERA, J.G.H. 2009. Concentración y combustión de vinazas. Ministerio de Gobierno y Justicia de la Provincia de Tucumán, Secretaría de Estado de Gobierno y Justicia, Subsecretaria de Asuntos Técnicos.

ROMERO SALAMANCA, C. A. 2008. Efecto de las fuentes orgánicas obtenidas de los subproductos agroindustriales de la caña de azúcar (Saccharum officinarum) y el plátano (Musa sp) sobre la actividad microbiana y enzimática del suelo. Tesis de Maestría, Universidad Nacional de Colombia (sede Palmira).

SCANDALIARIS, J., N. C. DANTUR \& A. LAZARTE, 1985. Posibilidad de utilizar vinazas en el cultivo de la caña de azúcar. Avance Agroindustrial 5: 16-18.

SUBIRÓS, J. F. \& E. MOLINA. 1992. Efectos de la aplicación de vinazas en la producción de caña de azúcar en las características químicas de un inceptisol de Guanacaste, Costa Rica. Agronomía Costarricense 16: 55-60.

URBANO TERRÓN, PEDRO 2002. Fertilización orgánica con vinazas de alcoholera. Vida Rural 155: 50-52.

Recibido 26 de mayo de 2014, aceptado el 21 de setiembre de 2014. 
